First publ. in: Inventiones Mathematicae 173 (2008), 1, pp. 1-6

The original publication is available at www.springerlink.com

\title{
A proof of the Pfister Factor Conjecture
}

\author{
Karim Johannes Becher \\ Universität Konstanz, FB Mathematik und Statistik, D203, 78457 Konstanz, Germany \\ (e-mail: becheramaths.ucd.ie)
}

\begin{abstract}
It is shown that any split product of quaternion algebras with orthogonal involution is adjoint to a Pfister form. This settles the Pfister Factor Conjecture formulated by D.B. Shapiro. A more general problem on decomposability for algebras with involution is posed and solved in the case where the algebra is equivalent to a quaternion algebra.
\end{abstract}

Let $K$ be a field of characteristic different from 2. By a $K$-algebra with involution we mean a pair $(A, \sigma)$ of a central simple $K$-algebra $A$ and a $K$-linear involution $\sigma: A \longrightarrow A$. Involutions of this kind are either orthogonal or symplectic.

A (regular) quadratic form $\varphi$ over $K$ can be understood as a pair $\left(V,\langle\cdot, \cdot\rangle_{\varphi}\right)$ of a finite-dimensional $K$-vector space $V$ and a nonsingular symmetric bilinear form $\langle\cdot, \cdot\rangle_{\varphi}: V \times V \longrightarrow K$. Let $\operatorname{Ad}(\varphi)$ denote the adjoint $K$-algebra with involution $\left(\operatorname{End}_{K}(V), \sigma\right)$ where $\sigma: \operatorname{End}_{K}(V) \longrightarrow \operatorname{End}_{K}(V)$ is determined by the condition that $\langle f(v), w\rangle_{\varphi}=\langle v, \sigma(f)(w)\rangle_{\varphi}$ for all $v, w \in V$ and $f \in \operatorname{End}_{K}(V)$.

Assigning to a quadratic form its adjoint algebra with involution yields a one-to-one correspondence between the similarity classes of quadratic forms over $K$ and the isomorphism classes of split $K$-algebras with orthogonal involution (see [7, p. 1]). This correspondence commutes with taking tensor products and with extension of the field of scalars. The notions of isotropy and hyperbolicity are defined for algebras with involution in such a way that, in particular, a quadratic form is anisotropic, isotropic, or hyperbolic if and only if its adjoint algebra with involution has the same property (see [2]).

Since Pfister forms play a key role in the theory of quadratic forms, it seems natural to look for a corresponding concept in the theory of algebras with involution. An $n$-fold Pfister form over $K$ is a quadratic form of the shape $\left\langle 1, a_{1}\right\rangle \otimes \cdots \otimes\left\langle 1, a_{n}\right\rangle$ over $K$, with $a_{1}, \ldots, a_{n} \in K^{\times}$. The algebra with involution adjoint to this form is isomorphic to $\bigotimes_{i=1}^{n} \operatorname{Ad}\left(\left\langle 1, a_{i}\right\rangle\right)$, which is 
a product of split $K$-quaternion algebras with involution. It is well-known that a quadratic form $\pi$ over $K$ is similar to an $n$-fold Pfister form if and only if $\operatorname{dim}(\pi)=2^{n}$ and $\pi_{L}$ is either anisotropic or hyperbolic for every field extension $L / K$. These facts together motivate the search for a similar characterization of products of quaternion algebras with involution within the class of $K$-algebras with involution.

Conjecture. Let $n \in \mathbb{N}$ and let $(A, \sigma)$ be a $K$-algebra with involution such that $\operatorname{deg}(A)=2^{n}$. There exist $K$-quaternion algebras with involution $\left(Q_{i}, \sigma_{i}\right)(1 \leq i \leq n)$ such that $(A, \sigma) \cong \bigotimes_{i=1}^{n}\left(Q_{i}, \sigma_{i}\right)$ if and only if, for every field extension $L / K$, the $L$-algebra with involution $(A, \sigma)_{L}$ is either anisotropic or hyperbolic.

In the case where $\sigma$ is orthogonal and $n \leq 3$, this is proven in $[1,(2.10)]$. In the split symplectic case, the conjecture holds trivially. In fact, if $A$ is split and $\sigma$ is symplectic, then $(A, \sigma)$ is hyperbolic and isomorphic to $\bigotimes_{i=1}^{n}\left(Q, \sigma_{i}\right)$ where $Q$ is the split quaternion algebra $M_{2}(K), \sigma_{1}$ is the canonical involution on $Q$, and $\sigma_{2}, \ldots, \sigma_{n}$ are arbitrary orthogonal involutions on $Q$.

In the case where $A$ is split and $\sigma$ is orthogonal, one has essentially to decide whether the decomposability of $(A, \sigma)$ into a product of $n$ quaternion algebras with orthogonal involution yields that $(A, \sigma) \cong \operatorname{Ad}(\pi)$ for some $n$-fold Pfister form $\pi$ over $K$. In [13, Chap. 9], Shapiro shows the equivalence of this problem with a conjecture on spaces of similarities of quadratic forms, which he had considered in his thesis and a series of papers around 1975 (see [13] for references) and called the Pfister Factor Conjecture, and to which he had obtained a solution in the cases where $n \leq 5$, and also for arbitrary $n$ over particular ground fields including number fields. After in [13] Shapiro gave a reformulation of the problem in terms of split products of quaternion algebras with involution, different proofs for $n \leq 5$ were given in [12], [14], and [1]. Recently, a proof of the case $n=6$ was obtained in [6, Sect. 10].

Theorem 1 confirms the above conjecture in the split orthogonal case and thus establishes the Pfister Factor Conjecture in full generality. The conjecture formulated above is in fact confirmed in the more general case where $A$ is Brauer equivalent to a quaternion algebra. This is done by the corollary for the symplectic case and by Theorem 2 for the orthogonal case.

The general reference for the theory of algebras with involution is [7]. A standard reference for the theory of quadratic forms over fields is [8].

Let $W K$ denote the Witt ring of $K$. For a quaternion algebra $Q$ over $K$ we denote by $K(Q)$ the function field of the conic defined by the pure part of the norm form of $Q$. Note that $K(Q)$ is a generic splitting field for $Q$.

Proposition. Let $(A, \sigma)$ be an algebra with orthogonal involution over $K$. Assume that $A$ is Brauer equivalent to a quaternion algebra $Q$ and that $(A, \sigma)_{K(Q)}$ is adjoint to a Pfister form over $K(Q)$. Then $(A, \sigma)_{K(Q)}$ is adjoint to $\psi_{K(Q)}$ for some Pfister form $\psi$ defined over $K$. 
Proof. Let $\pi$ be a Pfister form over $K(Q)$ and assume that $(A, \sigma)_{K(Q)}$ is adjoint to $\pi$. Recall that the extension $K(Q) / K$ is excellent (cf. [5, Appendix II] or [11]). By [5, Proposition 2.10], in order to show that $\pi$ can be defined as a Pfister form over $K$, it is sufficient to show that $\pi$ represents a class in the image of the natural map $W K \longrightarrow W K(Q)$ defined by scalar extension. This image is described by an exact sequence (cf. [10, Theorem 6b] or [3, Lemma 3.1]) as the kernel of the product of all the second residue maps $\partial_{v}^{2}: W K(Q) \longrightarrow W \kappa_{v}$ where $v$ is any $K$-valuation of $K(Q)$ with residue field $\kappa_{v}$.

Let $v$ be an arbitrary $K$-valuation on $K(Q)$ with residue field $\kappa_{v}$. We need to show that $\partial_{v}^{2}(\pi)=0$ in $W \kappa_{v}$. Since $Q$ is defined over $K$ and splits over $K(Q)$, a standard argument shows that $Q$ also splits over $\kappa_{v}$. Let $M$ denote the completion of $K(Q)$ with respect to $v$. As $\kappa_{v}$ is still the residue field of $v$ on $M$, one has $\partial_{v}^{2}(\pi)=\partial_{v}^{2}\left(\pi_{M}\right)$ in $W \kappa_{v}$. Let $L$ be the relative algebraic closure of $K$ in $M$. Then $L / K$ embeds naturally into $\kappa_{v} / K$, and $\kappa_{v} / L$ is a finite purely inseparable extension. As $L$ contains $K$ and is therefore of characteristic different from 2, it follows that the extension $\kappa_{v} / L$ is of odd degree. Therefore $Q$ already splits over $L$. Let $\rho$ be a quadratic form over $L$ such that $(A, \sigma)_{L} \cong \operatorname{Ad}(\rho)$. Then $\operatorname{Ad}\left(\rho_{M}\right) \cong(A, \sigma)_{M} \cong \operatorname{Ad}\left(\pi_{M}\right)$, so $\rho_{M}$ and $\pi_{M}$ are similar. As $\rho$ is defined over $L$, one has $\partial_{v}^{2}\left(\rho_{M}\right)=0$. It follows that one of $\partial_{v}^{1}(\pi)$ and $\partial_{v}^{2}(\pi)$ is trivial. But as $\pi$ is a Pfister form, $\partial_{v}^{2}(\pi)$ is either trivial or a scalar multiple of $\partial_{v}^{1}(\pi)$. Thus $\partial_{v}^{2}(\pi)=0$ in any case.

Lemma. Let $(A, \sigma)$ be an algebra with orthogonal involution over $K$. Assume that $A$ is Brauer equivalent to a quaternion algebra $Q$. Then $(A, \sigma)$ is hyperbolic if and only if $(A, \sigma)_{K(Q)}$ is hyperbolic.

Proof. This follows from the result proven independently in [4] and in [9, Proposition 3.3].

Theorem 1. Let $n \in \mathbb{N}$ and let $\left(Q_{1}, \sigma_{1}\right), \ldots,\left(Q_{n}, \sigma_{n}\right)$ be $K$-quaternion algebras with involution. If $\bigotimes_{i=1}^{n}\left(Q_{i}, \sigma_{i}\right)$ is a split $K$-algebra with orthogonal involution, then it is adjoint to a Pfister form.

Proof. If the involution of $\bigotimes_{i=1}^{n}\left(Q_{i}, \sigma_{i}\right)$ is orthogonal, then the number of symplectic factors $\left(Q_{i}, \sigma_{i}\right)$ is even. Any product of two quaternion algebras with symplectic involution is isomorphic to a product of two quaternion algebras with orthogonal involution. One may therefore assume that all factors in the product are orthogonal.

For $n=1$ the statement is trivial. We proceed by induction on $n$. Assume that the statement holds for $n$. Let $\left(Q_{1}, \sigma_{1}\right), \ldots,\left(Q_{n+1}, \sigma_{n+1}\right)$ be $K$-quaternion algebras with orthogonal involution such that $\bigotimes_{i=1}^{n+1} Q_{i}$ is split. Let $(A, \sigma)=\bigotimes_{i=1}^{n}\left(Q_{i}, \sigma_{i}\right)$ and $(Q, \tau)=\left(Q_{n+1}, \sigma_{n+1}\right)$. Then $A$ is Brauer equivalent to $Q$, and there exists a quadratic form $\varphi$ over $K$ such that $(A, \sigma) \otimes(Q, \tau) \cong \mathrm{Ad}(\varphi)$. By the induction hypothesis, $(A, \sigma)_{K(Q)}$ is adjoint to a Pfister form. The proposition shows that $(A, \sigma)_{K(Q)} \cong \operatorname{Ad}\left(\psi_{K(Q)}\right)$ for an $n$-fold Pfister form $\psi$ defined over $K$. If $(A, \sigma)$ is hyperbolic, then 
also $(A, \sigma) \otimes(Q, \tau)$ is hyperbolic and $\varphi$ is the hyperbolic $(n+1)$-fold Pfister form. Assume now that $(A, \sigma)$ is not hyperbolic. By the lemma, then $(A, \sigma)_{K(Q)}$ is not hyperbolic. So, the Pfister form $\psi_{K(Q)}$ is not hyperbolic, and therefore anisotropic.

Let $L=K(\psi)$. Then $\psi_{L(Q)}$ is an isotropic Pfister form, thus hyperbolic. Hence $(A, \sigma)_{L(Q)} \cong \operatorname{Ad}\left(\psi_{L(Q)}\right)$ is hyperbolic. By the lemma, this implies that $(A, \sigma)_{L}$ is hyperbolic. Therefore $(A, \sigma)_{L} \otimes_{L}(Q, \tau)_{L}$ is hyperbolic. Since this product is adjoint to $\varphi_{L}$, this shows that $\varphi$ becomes hyperbolic over $L=K(\psi)$. As $\operatorname{dim}(\psi)=2^{n}$ and $\operatorname{dim}(\varphi)=2^{n+1}$, using [8, Chap. X, Theorem 4.11] it follows that the anisotropic part of $\varphi$ is isometric to $\beta \otimes \psi$ for some quadratic form $\beta$ over $K$ with $\operatorname{dim}(\beta) \leq 2$. If $\operatorname{dim}(\beta)=0$ then $\varphi$ is the hyperbolic $(n+1)$-fold Pfister form. If $\operatorname{dim}(\beta)=2$, then $\varphi$ is similar to an $(n+1)$-fold Pfister form $\pi$ and $(A, \sigma) \cong \operatorname{Ad}(\varphi) \cong \operatorname{Ad}(\pi)$.

If $\operatorname{dim}(\beta)=1$ then, as $\psi_{K(Q)}$ is anisotropic, $\varphi_{K(Q)}$ is isotropic but not hyperbolic, thus not similar to a Pfister form. However, there exists $d \in K^{\times}$ such that $(Q, \tau)_{K(Q)} \cong \mathrm{Ad}\left(\langle 1,-d\rangle_{K(Q)}\right)$, and then $(A, \sigma)_{K(Q)} \otimes_{K(Q)}(Q, \tau)_{K(Q)}$ is adjoint to the Pfister form $(\psi \otimes\langle 1,-d\rangle)_{K(Q)}$, which therefore is similar to $\varphi_{K(Q)}$. Therefore $\operatorname{dim}(\beta) \neq 1$, and the proof is complete.

As a consequence, the different versions of the Pfister Factor Conjecture formulated by Shapiro in [13] all hold, for in [13, Chap. 9] it is shown that they are equivalent to the statement of Theorem 1.

Corollary. Let $Q$ be a quaternion algebra over $K$. Let $(A, \sigma)$ be a central simple $K$-algebra with symplectic involution such that $A$ is Brauer equivalent to $Q$ and $\operatorname{deg}(A)=2^{n}$ with $n \in \mathbb{N}$. Let $\gamma$ denote the canonical involution of $Q$. Then the following are equivalent:

(i) $(A, \sigma)$ is a product of quaternion algebras with involution.

(ii) $(A, \sigma) \otimes(Q, \gamma)$ is adjoint to a Pfister form.

(iii) $(A, \sigma) \cong(Q, \gamma) \otimes \operatorname{Ad}(\rho)$ for some Pfister form $\rho$ over $K$.

(iv) For any field extension $L / K,(A, \sigma)_{L}$ is either anisotropic or hyperbolic.

Proof. Let $\pi$ denote the norm form of $Q$. Then $(Q, \gamma) \otimes(Q, \gamma) \cong \operatorname{Ad}(\pi)$. Note that $(A, \sigma) \cong(Q, \gamma) \otimes \operatorname{Ad}(\vartheta)$ for a quadratic form $\vartheta$ over $K$ which represents 1 , and that $(A, \sigma)$ is isotropic or hyperbolic if and only if the same holds for the quadratic form $\pi \otimes \vartheta$ over $K$.

$(i \Rightarrow i i)$ This is immediate from Theorem 1 .

(ii $\Rightarrow$ iii $)$ Assume that $(A, \sigma) \otimes(Q, \gamma) \cong \operatorname{Ad}(\psi)$ for a Pfister form $\psi$ over $K$. As $(Q, \gamma)_{K(Q)}$ is hyperbolic, $\psi_{K(}(Q)$ is hyperbolic. By [8, Chap. X, Corollary 4.13], then $\psi \cong \pi \otimes \rho$ for some Pfister form $\rho$ over $K$. On the other hand, $(A, \sigma) \cong(Q, \gamma) \otimes \operatorname{Ad}(\vartheta)$ yields that $\operatorname{Ad}(\psi) \cong(A, \sigma) \otimes(Q, \gamma) \cong$ $\operatorname{Ad}(\pi \otimes \vartheta)$. Since $\psi$ is a Pfister form and $\pi \otimes \vartheta$ represents 1 , it follows that $\pi \otimes \vartheta \cong \psi \cong \pi \otimes \rho$. Therefore $\vartheta$ and $\rho$ extend to the same hermitian form over $(Q, \gamma)$, showing that $(A, \sigma) \cong(Q, \gamma) \otimes \operatorname{Ad}(\vartheta) \cong(Q, \gamma) \otimes \operatorname{Ad}(\rho)$.

(iii $\Rightarrow i)$ This is obvious. 
(iii $\Rightarrow$ iv) Assume that $(A, \sigma) \cong(Q, \gamma) \otimes \operatorname{Ad}(\rho)$ for a Pfister form $\rho$ over $K$. Let $L / K$ be a field extension. Then $(A, \sigma)_{L}$ is isotropic or hyperbolic if and only if the same holds for $(\pi \otimes \rho)_{L}$. Since $(\pi \otimes \rho)_{L}$ is a Pfister form, it is either anisotropic or hyperbolic, so the same is true for $(A, \sigma)_{L}$.

(iv $\Rightarrow i i$ ) Given a field extension $L / K,(A, \sigma)_{L}$ is isotropic or hyperbolic if and only if the same holds for $(A, \sigma)_{L} \otimes_{L}(Q, \gamma)_{L}$. Hence, if $(A, \sigma)_{L}$ is either anisotropic or hyperbolic for any field extension $L / K$, then $(A, \sigma) \otimes(Q, \gamma)$ is adjoint to a Pfister form, and thus is a product of quaternion algebras with involution.

Note that, if the quaternion algebra $Q$ is split, then $(A, \sigma)$ is hyperbolic and the equivalences in the corollary hold trivially.

Theorem 2. Let $(A, \sigma)$ be a central simple algebra with orthogonal involution over $K$. Assume that $A$ is Brauer equivalent to a quaternion algebra $Q$ over $K$ and that $\operatorname{deg}(A)=2^{n}$ with $n \geq 1$. Then the following are equivalent:

(i) $(A, \sigma)$ is isomorphic to a product of quaternion algebras with involution.

(ii) $(A, \sigma)_{K(Q)}$ is adjoint to a Pfister form.

(iii) $(A, \sigma) \cong(Q, \tau) \otimes \operatorname{Ad}(\rho)$ for some orthogonal involution $\tau$ on $Q$ and some $(n-1)$-fold Pfister form $\rho$ over $K$.

(iv) For any field extension $L / K,(A, \sigma)_{L}$ is either anisotropic or hyperbolic.

Proof. $(i) \Rightarrow$ (ii) This follows from Theorem 1 .

(ii) $\Rightarrow$ (iv) Assume that $(A, \sigma)_{K(Q)}$ is adjoint to a Pfister form. Let $L / K$ be a field extension such that $(A, \sigma)_{L}$ is not hyperbolic. By the lemma, then $(A, \sigma)_{L(Q)}$ is not hyperbolic. Since $L(Q)$ is an extension of $K(Q)$, the assumption implies that $(A, \sigma)_{L(Q)}$ is anisotropic. Thus $(A, \sigma)_{L}$ is anisotropic.

(iv) $\Rightarrow$ (ii) Let $\varphi$ be a quadratic form over $K(Q)$ with $(A, \sigma)_{K(Q)} \cong$ $\operatorname{Ad}(\varphi)$. Then $\operatorname{dim}(\varphi)=\operatorname{deg}(A)=2^{n}$. With the assumption of $(i v)$ it follows that $\varphi_{L}$ is anisotropic or hyperbolic for any field extension $L / K(Q)$. Therefore $\varphi$ is similar to a Pfister form $\pi$ over $K(Q)$, and then $(A, \sigma)_{K(Q)} \cong$ $\operatorname{Ad}(\varphi) \cong \operatorname{Ad}(\pi)$.

(ii) $\Rightarrow$ (iii) Assume that $(A, \sigma)_{K(Q)}$ is adjoint to a Pfister form. By the proposition, then $(A, \sigma)_{K(Q)} \cong \mathrm{Ad}\left(\pi_{K(Q)}\right)$ for an $n$-fold Pfister form $\pi$ over $K$. Let $\gamma$ denote the canonical involution on $Q$. Then $(A, \sigma)$ is adjoint to a skew-hermitian form $h$ of rank $2^{n-1}$ over $(Q, \gamma)$. Let $\delta \in Q^{\times}$be an element represented by $h$. Then $\delta$ is a pure quaternion. Let $d=\delta^{2} \in K^{\times}$. Note that $(A, \sigma)_{K(\sqrt{ } d)}$ is split and isotropic. As (ii) implies (iv), it follows that $(A, \sigma)_{K(\sqrt{ } d)}$ is hyperbolic. Since $(A, \sigma)_{K(\sqrt{ } d)} \cong \operatorname{Ad}\left(\pi_{K(\sqrt{ } d)}\right)$, then $\pi_{K(\sqrt{ } d)}$ is hyperbolic. Using [8, Chap. X, Theorem 1.5] it follows that $\pi \cong\langle 1,-d\rangle \otimes \rho$ over $K$ for some $(n-1)$-fold Pfister form $\rho$.

Let now $\tau$ denote the unique orthogonal involution on $Q$ with $\tau(\delta)=-\delta$. By [2, Proposition 3.4], $A$ contains an $\sigma$-invariant subalgebra isomorphic 
to $(Q, \tau)$. The centraliser of this subalgebra then is split and also $\sigma$-invariant. Then $(A, \sigma) \cong(Q, \tau) \otimes \operatorname{Ad}(\vartheta)$ where $\vartheta$ is a quadratic form over $K$ which represents 1 . Then $(A, \sigma)$ is adjoint to the hermitian form over $(Q, \tau)$ obtained from $\vartheta$ by extending scalars from $K$ to $Q$. Since $(Q, \tau)_{K(Q)}$ is adjoint to the quadratic form $\langle 1,-d\rangle$ over $K(Q)$, it follows that $(A, \sigma)_{K(Q)} \cong \operatorname{Ad}\left(\varphi_{K(Q)}\right)$ for the quadratic form $\varphi=\langle 1,-d\rangle \otimes \vartheta$ defined over $K$. Hence, $\varphi_{K(Q)}$ is similar to the Pfister form $\pi_{K(Q)}$. As $\varphi_{K(Q)}$ represents 1 , it follows that $\varphi_{K(Q)} \cong \pi_{K(O)}$. Therefore, the two hermitian forms over $(Q, \tau)$ obtained from $\rho$ and from $\vartheta$ by extending scalars from $K$ to $Q$ become isometric over $(Q, \tau)_{K(Q)}$ and thus, by the result proven in [4] and in [9, Proposition 3.3], they are already isometric over $(Q, \tau)$. Therefore $(A, \sigma) \cong(Q, \tau) \otimes \operatorname{Ad}(\rho)$.

$($ iii $) \Rightarrow($ i $)$ This is obvious.

Acknowledgements The author wishes to express his gratitude to Skip Garibaldi, Detlev Hoffmann, Alexander Prestel, Anne Quéguiner-Mathieu, Jean-Pierre Tignol, and Thomas Unger for their support in developing the ideas contained in this article.

\section{References}

1. Bayer-Fluckiger, E., Parimala, R., Quéguiner-Mathieu, A.: Pfister involutions. Proc. Indian Acad. Sci., Math. Sci. 113, 365-377 (2003)

2. Bayer-Fluckiger, E., Shapiro, D.B., Tignol, J.-P.: Hyperbolic involutions. Math. Z. 214, $461-476$ (1993)

3. Colliot-Thélène, J.-L., Sujatha, R.: Unramified Witt groups of real anisotropic quadrics. In: $K$-Theory and Algebraic Geometry: Connections with Quadratic Forms and Division Algebras (Santa Barbara, CA, 1992). Proc. Symp. Pure Math., vol. 58, Part 2, pp. 127 147. Am. Math. Soc., Providence, RI (1995)

4. Dejaiffe, I.: Formes antihermitiennes devenant hyperboliques sur un corps de déploiement. C. R. Acad. Sci., Paris, Sér. I, Math. 332, 105-108 (2001)

5. Elman, R., Lam, T.Y., Wadsworth, A.R.: Amenable fields and Pfister extensions. Proc. Conf. Quadratic Forms, Kingston 1976. Queen's Pap. Pure Appl. Math. 46, 445492 (1977) (With an appendix "Excellence of $F(\varphi) / F$ for 2-fold Pfister forms" by J.K. Arason)

6. Garibaldi, S., Parimala, R., Tignol, J.-P.: Discriminant of symplectic involutions. Pure Appl. Math. Q. (to appear)

7. Knus, M.-A., Merkurjev, A.S., Rost, M., Tignol, J.-P.: The Book of Involutions. Colloq. Publ., Am. Math. Soc., vol. 44. Am. Math. Soc., Providence, RI (1998)

8. Lam, T.Y.: Introduction to quadratic forms over fields. Grad. Stud. Math., vol. 67. Am. Math. Soc., Providence, RI (2005)

9. Parimala, R., Sridharan, R., Suresh, V.: Hermitian analogue of a theorem of springer. J. Algebra 243, 780-789 (2001)

10. Pfister, A.: Quadratic lattices in function fields of genus 0. Proc. London Math. Soc 66, 257-278 (1993)

11. Rost, M.: On quadratic forms isotropic over the function field of a conic. Math. Ann. 288, 511-513 (1990)

12. Serhir, A., Tignol, J.-P.: The discriminant of a decomposable symplectic involution. J. Algebra 273, 601-607 (2004)

13. Shapiro, D.B.: Compositions of Quadratic Forms. de Gruyter Expo. Math., vol. 33. Walter de Gruyter, Berlin (2000)

14. Sivatski, A.S.: Applications of Clifford algebras to involutions and quadratic forms Commun. Algebra 33, 937-951 (2005) 